\title{
Kl3 FORM FACTORS WITH NA48/2 AND NA62 STATUS*
}

\author{
RoBerto PiAndANi \\ on behalf of the NA48/2 and NA62 collaborations ${ }^{\dagger}$ \\ University of Pisa and INFN, Pisa, Italy
}

(Received July 30, 2018)

The NA48/2 experiment at CERN collected a very large sample of charged kaon decays into multiple final states. These data allow measurements related to QCD. We obtained our final measurement of the charged kaon semileptonic decays form factors based on 4.28 million $\mathrm{Ke} 3$ and 2.91 million $K \mu 3$ selected decays, with the smallest uncertainty for $K e 3$ and a competitive result for $K \mu 3$ and leading to the most precise combined $K l 3$ result coming from the kaon sector that reduces the form factor uncertainty of $\left|V_{u s}\right|$. The NA62 experiment at CERN SPS is designed to measure the branching ratio of the $K^{+} \rightarrow \pi^{+} \nu \bar{\nu}$ decay with $10 \%$ precision. $K \rightarrow \pi \nu \bar{\nu}$ is one of the theoretically cleanest meson decay where to look for indirect effects of new physics complementary to LHC searches. NA62 took data in 2015-2017; the analysis of a partial data set allows to reach the Standard Model sensitivity. The status of the experiments will be presented.

DOI:10.5506/APhysPolBSupp.11.617

\section{The $K^{ \pm} \rightarrow \mu^{ \pm} \pi^{0} \nu_{\mu}$ and $K^{ \pm} \rightarrow e^{ \pm} \pi^{0} \nu_{e}$ form factors}

The hadronic matrix element of semileptonic kaon decays $\left(K^{ \pm} l 3, l=\right.$ $e, \mu)$ is described by two dimensionless vector form factors $f_{ \pm}(t)$, which depend on the squared four-momentum transferred to the lepton system, $t=\left(p_{K}-p_{\pi}\right)^{2}$. In the matrix element $f_{-}$is multiplied by the lepton mass and, therefore, its contribution can be neglected in $K e 3$ decays. In addition to the two vector form factors, also a scalar form factor exists $\left(f_{0}\right)$. By construction $f_{0}(0)=f_{+}(0)$ and since $f_{+}(0)$ is not directly measurable, it is

* Presented at "Excited QCD 2018", Kopaonik, Serbia, March 11-15, 2018.

$\dagger$ Co-authors are listed at the end of this paper. 
customary to normalize to this quantity all the form factors so that

$$
\bar{f}_{+}(t)=\frac{f_{+}(t)}{f_{+}(0)}, \quad \bar{f}_{0}(t)=\frac{f_{0}(t)}{f_{+}(0)}, \quad \bar{f}_{+}(0)=\bar{f}_{0}(0)=1 .
$$

There exist many parametrizations of the $K l 3$ form factors in the literature, a widely known and most used is the Taylor expansion

$$
\bar{f}_{+, 0}(t)=1+\lambda_{+, 0}^{\prime} \frac{t}{m_{\pi^{ \pm}}^{2}}+\frac{1}{2} \lambda_{+, 0}^{\prime \prime}\left(\frac{t}{m_{\pi^{ \pm}}^{2}}\right)^{2},
$$

where $\lambda_{+, 0}^{\prime}$ and $\lambda_{+, 0}^{\prime \prime}$ are the slope and the curvature of the form factors, respectively. A second parametrization is present, this model, applying physical constraints, reduces to one the number of parameters used. A typical example is the pole one

$$
\bar{f}_{+, 0}(t)=\frac{M_{\mathrm{V}, \mathrm{S}}^{2}}{M_{\mathrm{V}, \mathrm{S}}^{2}-t},
$$

where the dominance of a single resonance is assumed and the corresponding pole mass $M_{\mathrm{V}, \mathrm{S}}$ is the only free parameter. A third parametrization exists, the dispersive parametrization [1]

$$
\begin{aligned}
\bar{f}_{+}(t) & =\exp \left[\left(\Lambda_{+}+H(t)\right) t / m_{\pi}^{2}\right], \\
\bar{f}_{0}(t) & =\exp \left[(\ln (C)-G(t)) t /\left(m_{K}^{2}-m_{\pi}^{2}\right)\right] .
\end{aligned}
$$

\subsection{Event selection and final background rejection}

The NA48/2 experiment and beam is described in [2]. The data selection requires one charged track in the DCHs and a time coincidence with at least two clusters in the electromagnetic calorimeter (the two $\gamma$ from the $\pi^{0}$ decay). Other requirements applied to the charged track are: a good reconstructed decay vertex inside the decay region and different conditions in the momentum depending on the track type $(p>5 \mathrm{GeV} / c$ for the electron, $p>10 \mathrm{GeV} / c$ for the muon). The electron identification is performed asking $E / p>0.9$, where $E$ is the energy deposited in the electromagnetic calorimeter and $p$ is the momentum measured in the spectrometer, and no signal in time in the MUV system. The muon identification is performed asking an associated hit in time in the MUV system and $E / p<0.9$. The background contribution has been estimated using the Monte Carlo. For $K e 3$, the background from $K^{ \pm} \rightarrow \pi^{ \pm} \pi^{0}$ has a significant contribution. In order to remove it from the final sample, a cut in the $P_{t}(\nu) \geqslant 0.03 \mathrm{GeV} / c$ of the event is applied, the final amount is less than $0.027 \%$. For $K \mu 3$ selection, essential background may come from $K^{ \pm} \rightarrow \pi^{ \pm} \pi^{0}$ decays with a following $\pi^{ \pm} \rightarrow \mu^{ \pm} \nu$. The final contamination is reduced to $0.0264 \%$ cutting in $m\left(\pi^{ \pm} \pi^{0}\right)$ and $m\left(\mu^{ \pm} \nu\right)$. 


\subsection{Preliminary form factors results}

The final statistics of selected data is $4.28 \times 10^{6}$ events for $K^{ \pm} e 3$, and $2.91 \times 10^{6}$ events for $K^{ \pm} \mu 3$. To extract the form factors, an events-weighting fit is performed in $5 \times 5 \mathrm{MeV}$ cells in the Dalitz plot of $E_{\pi^{0}}$ versus $E_{l}$ energies, computed in the kaon rest frame. Combining the $K \mu 3$ and $K e 3$ samples, the results for the parameters of the parametrization based on Taylor expansion are

$$
\begin{aligned}
& \lambda_{+}^{\prime}=23.35 \pm 0.75 \text { (stat.) } \pm 1.23 \text { (syst.) } \\
& \lambda_{+}^{\prime \prime}=1.73 \pm 0.29 \text { (stat.) } \pm 0.41 \text { (syst.) } \\
& \lambda_{0}^{\prime}=14.90 \pm 0.55 \text { (stat.) } \pm 0.80 \text { (syst.) } .
\end{aligned}
$$

For the pole parametrization, the results are

$$
M_{\mathrm{V}}=894.3 \pm 3.2 \text { (stat.) } \pm 5.4 \text { (syst.) }, \quad M_{\mathrm{S}}=1185.5 \pm 16.6 \text { (stat.) } \pm 35.5 \text { (syst.) } .
$$

For the dispersive parametrization, they are

$$
\begin{aligned}
\Lambda_{+} & =(22.67 \pm 0.18 \text { (stat.) } \pm 0.55 \text { (syst. })) \times 10^{-3}, \\
\ln |C| & =(189.12 \pm 4.91 \text { (stat.) } \pm 11.09 \text { (syst. })) \times 10^{-3} .
\end{aligned}
$$

\section{The $K \rightarrow \pi \nu \bar{\nu}$ physics motivation and present experimental status}

The rare decays $K^{+} \rightarrow \pi^{+} \nu \bar{\nu}$ and $K_{\mathrm{L}} \rightarrow \pi^{0} \nu \bar{\nu}$ are extremely attractive processes to study the physics of flavour because they both are exceptionally clean modes. The hard (quadratic) GIM mechanism is active; thus, these decays are dominated by short-distance dynamics. Moreover, the shortdistance amplitude is then governed by one single semileptonic operator whose hadronic matrix element can be determined experimentally by the semileptonic kaon decay; so the main hadronic uncertainties can be eliminated by experimental data. At the quark level, the two processes arise from the $s \rightarrow d \nu \bar{\nu}$ process, which in the Standard Model originates from a combination of the $Z$ penguin and a double $W$ exchange. The clearness of the two processes makes them a perfect test of the Standard Model in a way independent from the LHC physics. The theoretical predictions of the two Branching Ratios are [3]

$$
\begin{aligned}
\operatorname{Br}\left(K^{+} \rightarrow \pi^{+} \nu \bar{\nu}\right)_{\mathrm{SM}} & =(8.4 \pm 1.0) \times 10^{-11}, \\
\operatorname{Br}\left(K_{\mathrm{L}} \rightarrow \pi^{0} \nu \bar{\nu}\right)_{\mathrm{SM}} & =(3.4 \pm 0.6) \times 10^{-11} .
\end{aligned}
$$


The present experimental knowledge of the $K^{+} \rightarrow \pi^{+} \nu \bar{\nu}$ process is based on the observation of 7 events by the E787 and E949 collaborations at BNL. The result for the branching ratio is [4]

$$
\operatorname{Br}\left(K^{+} \rightarrow \pi^{+} \nu \bar{\nu}\right)_{\mathrm{EXP}}=\left(17.3_{-10.5}^{+11.5}\right) \times 10^{-11} .
$$

\subsection{The $K^{+} \rightarrow \pi^{+} \nu \bar{\nu}$ analysis}

The description of the NA62 experiment and beam is in [5]. The analysis of $5 \%$ of data collected in $2016\left(2.3 \times 10^{10}\right.$ kaons $)$ has been presented. The $K^{+} \rightarrow \pi^{+} \nu \bar{\nu}$ signature is one track in the initial (the kaon) and final state (the pion) with two missing neutrinos. The main kinematic variable is $m_{\text {miss }}^{2}=\left(P_{K}-P_{\pi}\right)^{2}$, where $P_{K}$ and $P_{\pi}$ are the 4-momenta of the kaon and pion respectively. The theoretical shapes of the $m_{\text {miss }}^{2}$ distribution for the main $K^{+}$background decay modes are compared to the signal in Fig. 1. The considered pion momentum range is between 15 and $35 \mathrm{GeV} / c$ to leave at least $40 \mathrm{GeV}$ of electromagnetic energy in the calorimeters in the case of $K^{+} \rightarrow \pi^{+} \pi^{0}$ decay. Two regions are considered in order to reduce the main background contamination using the kinematic: Region I between $K^{+} \rightarrow \mu^{+} \nu$ and $K^{+} \rightarrow \pi^{+} \pi^{0}$ and Region II between $K^{+} \rightarrow \pi^{+} \pi^{0}$ and $K^{+} \rightarrow \pi^{+} \pi^{+} \pi^{-}$. Each of the background processes requires a different rejection procedure depending on its kinematics and type of charged particle in the final state. The main requirements for the analysis are excellent kinematic reconstruction to reduce kinematic tails; precise timing to reduce the kaon mis-tagging probability; no extra in-time activity in all of the electromagnetic calorimeters to suppress $K^{+} \rightarrow \pi^{+} \pi^{0}$ decays with $\pi^{0} \rightarrow \gamma \gamma$; clear separation between $\pi / \mu / e$ tracks to suppress decays with $\mu^{+}$or $e^{+}$in

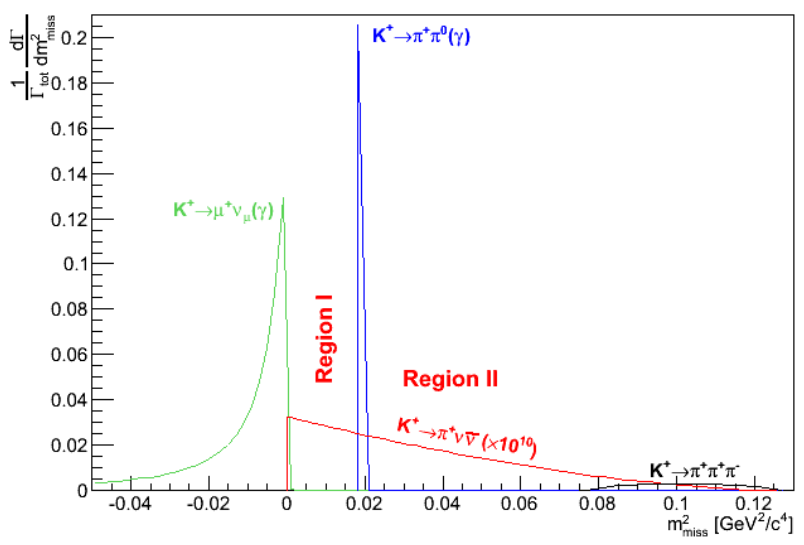

Fig. 1. Theoretical squared missing mass distribution of the main background and the signal (multiplied for a factor 10). 
the final state. Low multiplicity cuts in the downstream detectors are used to further suppress decays with multiple charged tracks in the final state. The parent kaon track is reconstructed in the GTK with 100 ps resolution; the daughter pion track is reconstructed in the STRAW. The RICH measure $\pi^{+}$time with resolution below $100 \mathrm{ps}$. The pion is associated in time to a KTAG kaon signal. The timing and the closest distance of approach between GTK and STRAW tracks allow a precise $K^{+}-\pi^{+}$matching. The kaon mis-tagging probability at $40 \%$ of nominal intensity is below $2 \%$, signal acceptance about $75 \%$. Reconstruction tails from $K^{+} \rightarrow \pi^{+} \pi^{0}, K^{+} \rightarrow \mu^{+} \nu$ and $K^{+} \rightarrow \pi^{+} \pi^{+} \pi^{-}$set the level of background in signal regions. To reduce it, signal regions are restricted to boxes within a 3D space, defined by (1) $m_{\text {miss }}^{2}$; (2) the same quantity computed using the momentum of the particle measured by the RICH under pion hypothesis rather than the straws $\left(m_{\text {miss }}^{2}\right.$ $(\mathrm{RICH}))$; (3) the same quantity computed replacing the 3-momentum of the kaon measured by the GTK with the nominal 3-momentum of the beam $\left(m_{\text {miss }}^{2}(\mathrm{No}-\mathrm{GTK})\right)$. Calorimeters and RICH separate $\pi^{+}, \mu^{+}$, and $e^{+}$. Remaining events after PID are primarily $K^{+} \rightarrow \pi^{+} \pi^{0}$. Photon rejection exploiting timing coincidences between charged pion and calorimetric deposits suppresses them further. A sample of $K^{+} \rightarrow \pi^{+} \pi^{0}$ from minimum bias is used for normalization. About $0.064 K^{+} \rightarrow \pi^{+} \nu \bar{\nu}$ events are expected over $2.3 \times 10^{10}$ kaon decays. Figure 2 shows the distribution of residual events

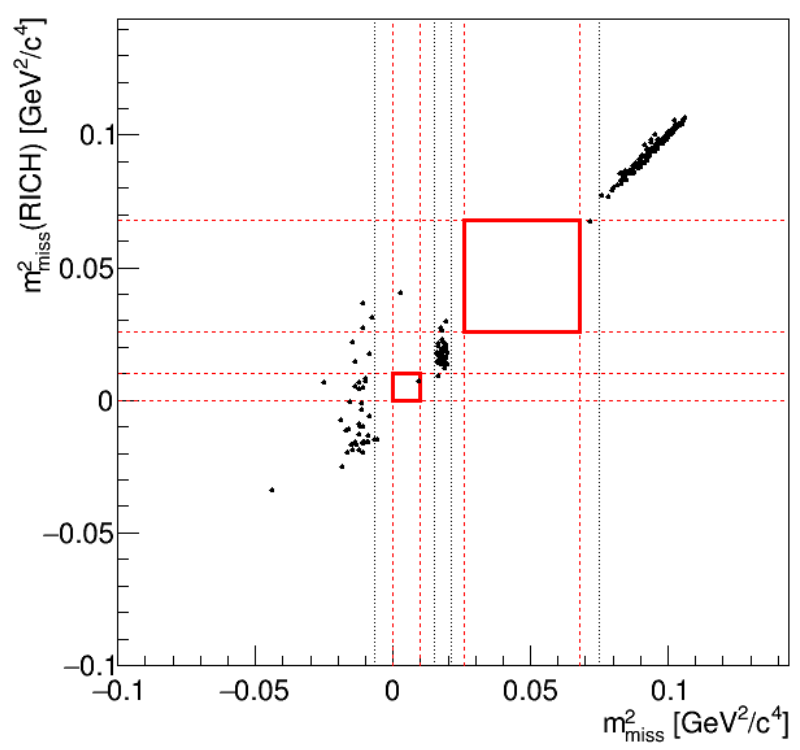

Fig. 2. Distribution in the $\left(m_{\text {miss }}^{2}(\mathrm{RICH}), m_{\text {miss }}^{2}\right)$ plane of $K^{+} \rightarrow \pi^{+} \nu \bar{\nu}$-triggered events passing the selection; signal regions (thick boxes) and lines defining background regions (light dashed lines) are drawn. 
in the $m_{\text {miss }}^{2}(\mathrm{RICH})$ versus $m_{\text {miss }}^{2}$ plane. Backgrounds from $K^{+} \rightarrow \pi^{+} \pi^{0}$, $K^{+} \rightarrow \mu^{+} \nu$ and $K^{+} \rightarrow \pi^{+} \pi^{+} \pi^{-}$are $0.024,0.011$ and 0.017 , respectively. They are estimated directly from events outside signal regions, with the measured kinematic tails used for extrapolation in signal regions. The analysis of the full 2016 sample is on-going together with an optimization of the selection to further reduce backgrounds and increase signal acceptance. No events are observed in signal regions, the event inside region 1 has $m_{\text {miss }}^{2}$ (no-GTK) outside the box.

\section{REFERENCES}

[1] V. Bernard, M. Oertel, E. Passemar, J. Stern, Phys. Rev. D 80, 034034 (2009).

[2] V. Fanti et al. [NA48 Collaboration], Nucl. Instrum. Methods Phys. Res. A 574, 433 (2007).

[3] A.J. Buras, D. Buttazzo, J. Girrbach-Noe, R. Knegjens, J. High Energy Phys. 1511, 033 (2015).

[4] S. Adler et al. [E949 and E787 collaborations], Phys. Rev. D 77, 052003 (2008).

[5] E. Cortina Gil et al. [NA62 Collaboration], JINST 12, P05025 (2017).

The NA48/2 and NA62 collaborations members:

R. Aliberti, F. Ambrosino, R. Ammendola, B. Angelucci, A. Antonelli, G. Anzivino, R. Arcidiacono, M. Barbanera, A. Biagioni, L. Bician, C. Biino, A. Bizzeti, T. Blazek, B. Bloch-Devaux, V. Bonaiuto, M. Boretto, M. Bragadireanu, D. Britton, F. Brizioli, M.B. Brunetti, D. Bryman, F. Bucci, T. Capussela, A. Ceccucci, P. Cenci, V. Cerny, C. Cerri, B. Checcucci, A. Conovalo, P. Cooper, E. Cortina Gil, M. Corvino, F. Costantini, A. Cotta Ramusino, D. Coward, G. D'Agostini, J. Dainton, P. Dalpiaz, H. Danielsson, N. De Simone, D. Di Filippo, L. Di Lella, N. Doble, B. Dobrich, F. Duval, V. Duk, J. Engelfried, T. Enik, N. Estrada-Tristan, V. Falaleev, R. Fantechi, V. Fascianelli, L. Federici, S. Fedotov, A. Filippi, M. Fiorini, J. Fry, J. Fu, A. Fucci, L. Fulton, E. Gamberini, L. Gatignon, G. Georgiev, S. Ghinescu, A. Gianoli, M. Giorgi, S. Giudici, F. Gonnella, E. Goudzovski, C. Graham, R. Guida, E. Gushchin, F. Hahn, H. Heath, T. Husek, O. Hutanu, D. Hutchcroft, L. Iacobuzio, E. Iacopini, E. Imbergamo, B. Jenninger, K. Kampf, V. Kekelidze, S. Kholodenko, G. Khoriauli, A. Khotyantsev, A. Kleimenova, A. Korotkova, M. Koval,V. Kozhuharov, Z. Kucerova, Y. Kudenko, J. Kunze, V. Kurochka, V. Kurshetsov, G. Lanfranchi, G. Lamanna, G. Latino, P. Laycock, C. Lazzeroni, M. Lenti, G. Lehmann Miotto, E. Leonardi, P. Lichard, L. Litov, R. Lollini, D. Lomidze, A. Lonardo, P. Lubrano, M. Lupi, N. Lurkin, D. Madigozhin, I. Mannelli, G. Mannocchi, A. Mapelli, F. Marchetto, R. Marchevski, 
S. Martellotti, P. Massarotti, K. Massri, E. Maurice, M. Medvedeva, A. Mefodev, E. Menichetti, E. Migliore, E. Minucci, M. Mirra, M. Misheva, N. Molokanova, M. Moulson, S. Movchan, M. Napolitano, I. Neri, F. Newson, A. Norton, M. Noy, T. Numao, V. Obraztsov, A. Ostankov, S. Padolski, R. Page, V. Palladino, C. Parkinson, E. Pedreschi, M. Pepe, M. Perrin-Terrin, L. Peruzzo, P. Petrov, F. Petrucci, R. Piandani, M. Piccini, J. Pinzino, I. Polenkevich, L. Pontisso, Yu. Potrebenikov, D. Protopopescu, M. Raggi, A. Romano, P. Rubin, G. Ruggiero, V. Ryjov, A. Salamon, C. Santoni, G. Saracino, F. Sargeni, V. Semenov, A. Sergi, A. Shaikhiev, S. Shkarovskiy, D. Soldi, V. Sougonyaev, M. Sozzi, T. Spadaro, F. Spinella, A. Sturgess, J. Swallow, S. Trilov, P. Valente, B. Velghe, S. Venditti, P. Vicini, R. Volpe, M. Vormstein, H. Wahl, R. Wanke, B. Wrona, O. Yushchenko, M. Zamkovsky, A. Zinchenko. 